\title{
Observer les déplacements dans la construction des scènes locales
}

Le cas de la musique metal en France

Observe the movements of the users in the construction of the local scenes

The case of heavy metal in France

Observar los desplazamientos en la construcción de las escenas locales

\section{El caso de la música metálica}

\section{Sophie Turbé}

\section{Numéro 57, automne 2014}

La notion de "scène ", entre sociologie de la culture et sociologie urbaine : genèse, actualités et perspectives

URI : https://id.erudit.org/iderudit/1035277ar

DOI : https://doi.org/10.7202/1035277ar

Aller au sommaire du numéro

Éditeur(s)

Athéna éditions

ISSN

0831-1048 (imprimé)

1923-5771 (numérique)

Découvrir la revue

Citer cet article

Turbé, S. (2014). Observer les déplacements dans la construction des scènes locales : le cas de la musique metal en France. Cahiers de recherche sociologique, (57), 97-113. https://doi.org/10.7202/1035277ar
Résumé de l'article

Quelles sont les limites géographiques d'une scène locale ? Comment ces acteurs agissent comme " agents performatifs » dans la perception de l'espace territorial recomposé par la musique ? Il s'agit donc de démontrer l'intérêt de sortir d'une vision en vase clos des scènes locales, pour s'intéresser de plus près aux déplacements de ceux qui les pratiquent (musiciens, publics), à la façon dont Guy Di Méo (1996) propose une géographie socio-culturelle des " territoires du quotidien".

La démonstration repose sur l'observation des déplacements des amateurs de musique metal issus de trois régions françaises, à savoir la Lorraine, le Nord-pas-de-Calais et les environs de Rennes et Nantes. L'étude s'appuie sur un matériel empirique composé de données quantitatives et qualitatives. 


\section{Observer les déplacements dans la construction des scènes locales Le cas de la musique metal en France}

SOPHIE TURBÉ

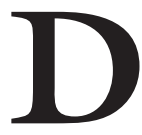
essiner les limites d'une scène locale semble une entreprise hasardeuse. Les travaux qui se lancent dans l'analyse d'une scène définissent en général le «local» en fonction de frontières préexistantes aux individus et à leurs pratiques, le plus souvent celles de la ville, de la région ou du pays ${ }^{1}$. La notion de frontière en tant que telle n'est donc pas particulièrement discutée dans le cadre des travaux en sociologie qui se consacrent à l'étude de scènes culturelles et, a fortiori, aux scènes musicales. Elle intéresse pourtant davantage les géographes qui, dans les travaux en géographie culturelle dans le sillage de ceux d'Armand Frémont ${ }^{2}$ et de Guy Di Méo ${ }^{3}$, ont interrogé la notion d'«espace vécu» pour comprendre de quelles façons les individus s'approprient l'espace dans le cadre de leurs pratiques quotidiennes et produisent des représentations sur ceux-ci. Pour Armand Frémont, l'«espace vécu» désigne «l'ensemble des

1. Fabien Hein, Le monde du rock. Ethnographie du réel, Paris, Irma/Séteun, 2006; Yves Raibaud, Territoires musicaux en région: l'émergence des musiques amplifiées en Aquitaine, Pessac, MSH d'Aquitaine, 2005; Jean-Pierre Augustin, Micheline Cassou-Mounat, «Les jeunes dans la ville: institutions de socialisation et différenciation spatiale dans la Communauté urbaine de Bordeaux», Annales de Géographie, vol. 102, n 573, 1993, p. 548-550; Gérôme Guibert, La production de la culture. Le cas des musiques amplifiées en France, Paris, Irma/Seteun, 2006; Samuel Balti, «Les musiques amplifiées et leur impact dans la structuration des territoires métropolitains: l'exemple des activités de diffusion dans l'agglomération toulousaine», Sud-Ouest Européen, n² 27, 2009.

2. Armand Frémont, La région, espace vécu, Paris, Flammarion, 1999.

3. Guy Di Méo, Les territoires du quotidien, Paris, L'Harmattan,1996. 
lieux fréquentés - par l'individu - mais aussi des interrelations qui s’y nouent et les valeurs psychologiques qui y sont projetées et perçues ${ }^{4}$ ». Appliqué au contexte des scènes musicales, Gérôme Guibert parle ainsi de «scène vécue ${ }^{5}$ » pour décrire les représentations mentales de la scène construites par ses pratiquants à partir de leurs expériences directes du territoire dans lequel s'inscrivent les rapports entre les acteurs du monde musical.

Si la notion de scène locale permet de territorialiser la musique en rattachant des pratiques culturelles à un espace vécu, il semble judicieux de s'intéresser plus en détails au territoire pratiqué par les usagers des scènes locales en observant leurs déplacements au sein d'un espace géographique aux contours imprécis. S'intéresser aux déplacements, et donc aux distances parcourues, implique ainsi obligatoirement de poser la question des frontières de la scène locale: à quel moment, ou à quel endroit, l'individu qui nourrit un sentiment d'appartenance à une scène locale aura l'impression de se trouver à l'intérieur de celle-ci ou bien d'être passé à l'extérieur?

Se déplacer loin pour assister à un concert, mais effectuer ce trajet régulièrement, amènent ainsi souvent les individus à se déplacer sur un vaste territoire et à franchir des frontières administratives tout en ayant le sentiment de rester à l'intérieur de leur espace de connaissance. Ces représentations mentales de la scène locale sont autant de «terrains de jeux» musicaux, qui peuvent être perçus par chacun de leurs pratiquants avec un point de vue singulier, en fonction de leur lieu d'habitation, et des moyens dont ils disposent, pour se rendre sur les lieux d'un concert. La perception d'une scène locale par ses usagers semble donc se construire d'abord à travers une unité de lieu, qui est au fondement de la notion de «local», mais aussi à travers une certaine forme de temporalité, dans le sens où ces usagers, lorsqu'ils se rendent sur les lieux d'un concert, ne considèrent l'événement comme étant de l'ordre de la scène locale que s'il s'agit de déplacements routiniers, que l'on effectue simplement au cours d'une soirée. Par exemple, le déplacement d'un individu résidant à Nantes pour assister à un concert à Paris et qui nécessitera une organisation spécifique comme, entre autres, un hébergement pour le soir même, ne pourra probablement plus, en fonction de cela, être considéré comme appartenant à la scène locale.

Du point de vue de ses acteurs, la scène musicale locale est ici considérée dans son acception la plus large. Elle est à la fois un ensemble de lieux, d'individus et de groupes d'individus (des groupes de musique, des

4. Armand Frémont, «Espace vécu et niveaux sociaux», L'espace vécu, Actes du colloque, Paris, CNRS-RCP n 354, 1976, p. 219.

5. Gérôme Guibert, «La notion de scène locale. Pour une approche renouvelée de l'analyse des courants musicaux», Stéphane Dorin (dir.), Sound Factory, Paris, Seteun, 2012, p. 109. 
intermédiaires organisationnels et médiatiques, des publics) qui collaborent ensemble, au sens où Howard Becker a théorisé la notion de «mondes de l'art ${ }^{6} »$, à la construction d'un territoire commun; mais aussi d'événements qui ponctuent le quotidien d'un espace vécu dans le temps et qui lui confèrent une historicité. Dans cette acception, la notion de scène locale semble étroitement liée à la notion d'expérience, en ce qu'il s'agit d'une abstraction vécue par ses pratiquants qui leur permet d'identifier un espace communautaire qui répond à un certain nombre de critères: la connaissance des lieux (les salles, mais aussi les villes, les villages, les itinéraires) ainsi que la connaissance des personnes (le fait de se retrouver entre amis, d'y croiser régulièrement les mêmes personnes, ce qui implique souvent le sentiment d'appartenir au même réseau relationnel). Il s'agit alors de territoires (relationnels ou géographiques) expérimentés, voire maîtrisés, qui ne représentent pas un inconnu pour les usagers. La dimension communautaire des scènes locales est par ailleurs perceptible dans la tendance qu'ont les pratiquants à la nommer (la «scène lorraine », la «scène nantaise », la «scène punk de Bordeaux », etc.) selon différentes échelles de points de vue, ou simplement lorsque les individus ont recours à l'expression «scène locale» dans leurs conversations du quotidien pour qualifier le «milieu» ou «l'écosystème» auquel ils prennent part, et qui révèle un sentiment d'identité collective.

Dans le cadre de cette contribution, il s'agit ainsi de comprendre, dans une approche contrastive, de quelles façons se construisent les territoires locaux recomposés par la musique. Pour cela, nous proposons de prendre le point de vue des usagers des scènes locales, qu'ils soient musiciens, organisateurs ou publics ${ }^{7}$ afin de raccorder «les éléments structuraux qui les charpentent et les espaces vécus qui s’en dégagent ${ }^{8} »$.

6. Howard Becker, Les mondes de l'art, Paris, Flammarion, 1988.

7. Seuls $21 \%$ des répondants au questionnaire ne sont «que» des publics. En effet, les publics des concerts de metal sont souvent eux-mêmes investis dans d'autres pratiques de production telles qu'un groupe, la participation à l'organisation de concerts, la rédaction de chroniques musicales ou encore la réalisation et la distributions d'affiches et dépliants.

8. Guy Di Méo, Introduction à la géographie sociale, Paris, Armand Colin, 2014, p. 43. 


\section{Construction de l'espace vécu des scènes locales}

\section{Caractéristiques de l'échantillon}

Les résultats statistiques exploités tout au long de cette contribution reposent sur les réponses à un questionnaire diffusé auprès des amateurs de metal issus de trois espaces régionaux français: le Nord-Pas-de-Calais, la Lorraine et l'Illeet-Vilaine/Loire-Atlantique9. Le questionnaire a été diffusé sur Internet (webzines, réseaux sociaux) grâce aux acteurs locaux (associations et médias spécialisés) entre février et juillet 2013. L'échantillon ainsi obtenu est proche en termes de caractéristiques des résultats de l'enquête française menée par Fabien Hein ${ }^{10}$. Tous les entretiens exploités dans le cadre de cet article proviennent par ailleurs d'enquêtés rencontrés par l'intermédiaire de ce questionnaire.

L'échantillon est ainsi composé de $85 \%$ d'hommes et de $15 \%$ de femmes, dont l'écart-type fait varier la moyenne d'âge de 19 à 33 ans. La faible représentativité des femmes au sein de l'échantillon, bien que particulièrement marquée ici, est notable dans de nombreuses études sur la musique metal ${ }^{11}$. Il s'agit en effet d'une pratique culturelle très masculinisée, mais dans laquelle les femmes sont néanmoins de plus en plus visibles ${ }^{12}$. Du point de vue socioprofessionnel, globalement, les amateurs de metal qui sont insérés dans la vie active ou dans un cycle d'étude dominent l'échantillon. Ainsi, les individus sans emploi ne représentent que $9 \%$ des amateurs interrogés ${ }^{13}$. Les catégories les plus représentées sont celle des employés (35\%), suivie des étudiants $(23 \%)^{14}$. Ce qui nous semble indiquer que les amateurs de metal interrogés disposent pour la plupart de ressources économiques qui leur permettent de se déplacer facilement sur le territoire.

Du point de vue de l'origine géographique des individus, l'échantillon se compose environ pour moitié ${ }^{15}$ d'amateurs vivant dans de grandes aires

9. 523 réponses exploitables ont été récoltées, dont 219 pour l'Ille-et-Vilaine/Loire-Atlantique, 185 pour la Lorraine et 119 pour le Nord-pas-de-Calais.

10. Fabien Hein, Hard rock heavy metal metal: Histoire, cultures et pratiquants, Paris, Irma/Seteun, 2003.

11. Robert Walser, Running with the devil: power, gender and madness in heavy metal music, Delaware, Wesleyan University Press, 1993; Deena Weinstein, Heavy Metal. The music and its culture, New York, Da Capo Press, 1991; Bettina Roccor, Heavy Metal. Die band. Die fans. Die gegner, München, Beck'sche Reihe, 1998.

12. F. Hein, op. cit, p. 178

13. Ce qui est légèrement inférieur à la moyenne nationale française en 2013, au moment de l'enquête (autour de $10,3 \%)$.

14. Puis des sans-emplois ( $9 \%)$, des cadres et professions intellectuelles supérieures (5\%) et des agriculteurs exploitants $(0,22 \%)$. L'échantillon ne comprend aucun individu à la retraite.

15. Les individus issus des départements de Loire-Atlantique et Ille-et-Vilaine sont $57 \%$ à vivre au sein des agglomérations de Rennes ou Nantes, $21 \%$ à vivre dans une ville de plus de 10000 habitants et $22 \%$ dans une ville de moins de 10000 habitants. Les individus de Lorraine sont $51 \%$ à vivre au sein des agglomérations de Nancy ou Metz, $20 \%$ dans une ville de plus de 10000 habitants et $29 \%$ dans une ville de moins de 10000 habitants. Les individus du Nord-Pas-de-Calais sont $47 \%$ à vivre au sein de l'agglomération lilloise, $36 \%$ à vivre dans une ville de plus de 10000 habitants et $17 \%$ dans une ville de moins de 10000 habitants. 
urbaines de plus de 100000 habitants, riches en lieux de sorties culturelles et dans lesquelles les transports en commun possèdent un réseau développé. Pour autant, trois amateurs interrogés sur quatre ${ }^{16}$ privilégient la voiture comme mode de transport pour se déplacer en concert et près de la moitié17 d'entre eux n'hésite pas à parcourir entre 50 et $150 \mathrm{~km}$ pour s'y rendre. Les réponses des amateurs de metal témoignent donc d'une grande mobilité. À cela s'ajoute également une fréquence importante de sorties en concert, puisque les individus de l'échantillon déclarent se rendre en moyenne à un concert de metal par mois ${ }^{18}$.

\section{Le noyau de la scène locale: les métropoles}

Les centres métropolitains concentrent la majeure partie des déplacements. Ce premier constat est flagrant lorsqu'on demande aux enquêtés quels sont les cinq derniers concerts auxquels ils ont assisté. Dans le cas des trois scènes locales étudiées, les déplacements sont ainsi très largement concentrés vers des villes de plus de 100000 habitants ${ }^{19}$ et en l'occurrence, les villes les plus importantes de chaque région concernée, concentrant une large population étudiante.

On retrouve ce principe de centralité au niveau des déplacements dans le Nord-Pas-de-Calais (Fig.l) où Lille, la capitale régionale, apparaît ainsi très nettement comme la première destination des publics de metal locaux (plus d'un déplacement sur trois). De même, en Lorraine (Fig.2), la ville de Nancy représente un quart des déplacements. En ce qui concerne l'Illeet-Vilaine/Loire-Atlantique (Fig.3), les déplacements se concentrent autour de deux pôles. Ainsi, parmi les deux villes les plus citées par les amateurs de cette région, $40 \%$ des déplacements ont eu lieu à Nantes et 12\% à Rennes. Ensemble, les deux villes représentent plus de la moitié des déplacements sur les cinq derniers concerts. Dans l'Ouest de la France, Rennes et Nantes sont deux métropoles attractives, situées à environ $100 \mathrm{~km}$ l'une de l'autre et chacune capitale de leur région respective, La Bretagne et le Pays de la Loire. Malgré leur séparation administrative, les deux villes entretiennent des rapports qui, bien qu'inégaux, semblent pourtant structurants du point de

16. Sur la totalité de l'échantillon: $77 \%$ des individus déclarent utiliser le plus souvent la voiture pour se rendre en concert de metal, $14 \%$ les transports en commun, $5 \%$ s'y rendent le plus souvent à pied et $4 \%$ utilisent d'autres moyens de transports (deux roues et engins à roulettes).

17. À la question "Combien de kilomètres parcourez-vous en moyenne pour assister à un concert?», $44 \%$ des individus de l'échantillon ont répondu entre 50 et $150 \mathrm{~km}, 42 \%$ moins de $50 \mathrm{~km}$ et $13 \%$ plus de $150 \mathrm{~km}$. Ces données changent d'une scène à l'autre: les amateurs lorrains sont par exemple ceux qui déclarent parcourir le plus de kilomètres ( $55 \%$ ont répondu entre 50 et $150 \mathrm{~km}$, contre $49 \%$ pour les amateurs d'llle-et-Vilaine/ Loire-Atlantique et $47 \%$ pour ceux du Nord-Pas-de-Calais).

18. La moyenne est de 6 concerts avec un écart moyen en plus ou en moins de 5,2.

19. Nancy: 105000 hab., Rennes: 209000 hab., Nantes: 291000 hab., Lille: 229000 hab. 


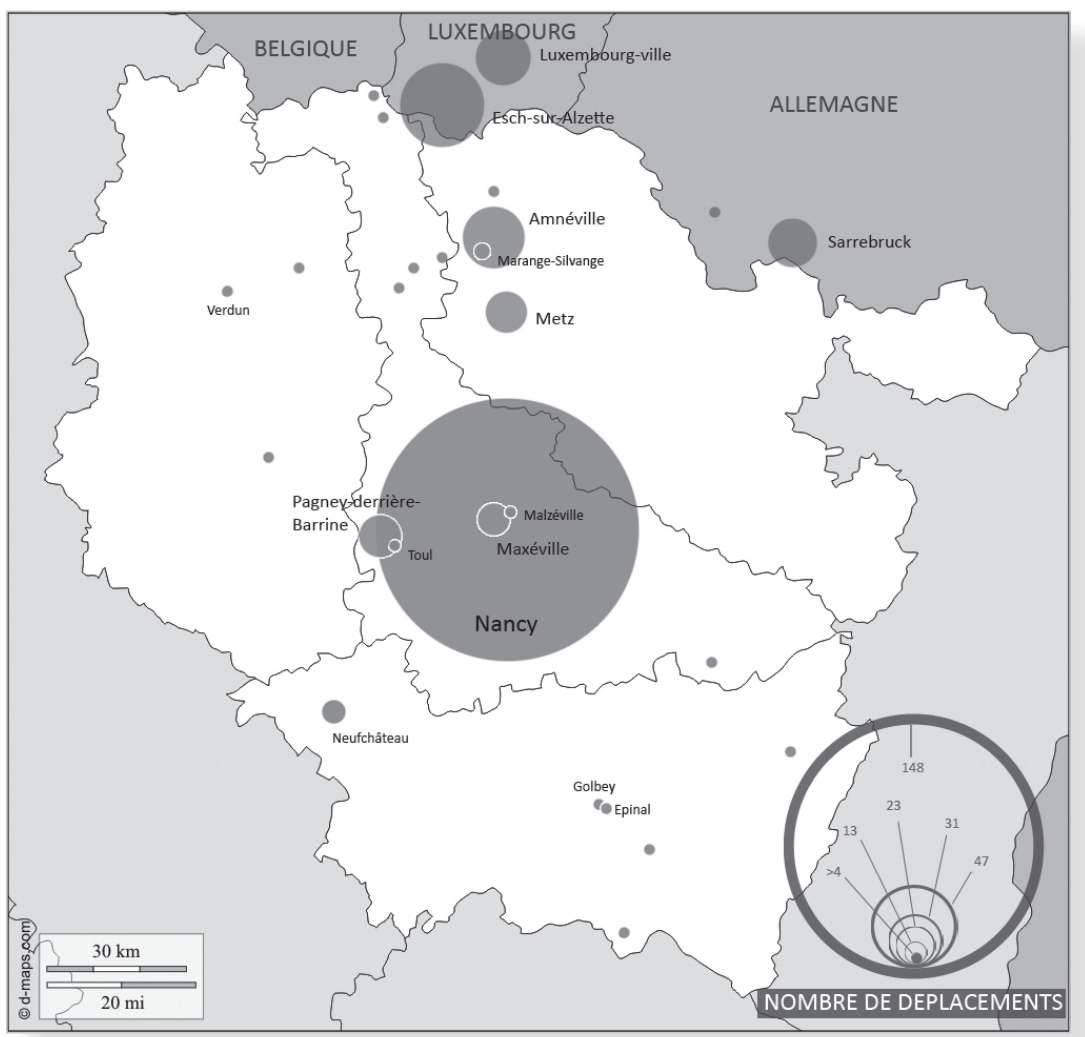

Figure 1

Nord-pas-de-Calais : déplacements au cours des 5 derniers concerts

vue de la scène metal locale. Les amateurs de metal rennais sont par exemple $30 \%$ à s'être rendus à Nantes au cours de leurs derniers déplacements. De leur côté, les amateurs nantais ne sont que $5 \%$ à avoir fait le chemin inverse. L'attractivité de la scène metal nantaise semble donc rayonner au-delà des frontières régionales et accaparer une partie du public rennais.

Bien que la scène metal rennaise soit dynamique, avec plus d'un concert organisé par semaine en moyenne, Nantes atteint le record de 2,5 concerts de metal organisés par semaine ${ }^{20}$, ce qui en fait la ville française la plus riche en soirées metal après Paris. Ce dynamisme est à imputer à l’hyper spécialisation de certaines de ses salles, parmi lesquelles Le Ferrailleur se distingue tout particulièrement. Il s'agit d'un bar-concert d'une capacité d'environ

20. D'après l'analyse de la programmation menée de juin 2012 à juin 2013 dans chacun des trois espaces géographiques étudiés: un recensement systématique des concerts de metal donnés au cours des 12 mois a été effectué par croisement des données entre les principaux agendas de concerts de metal en France. 


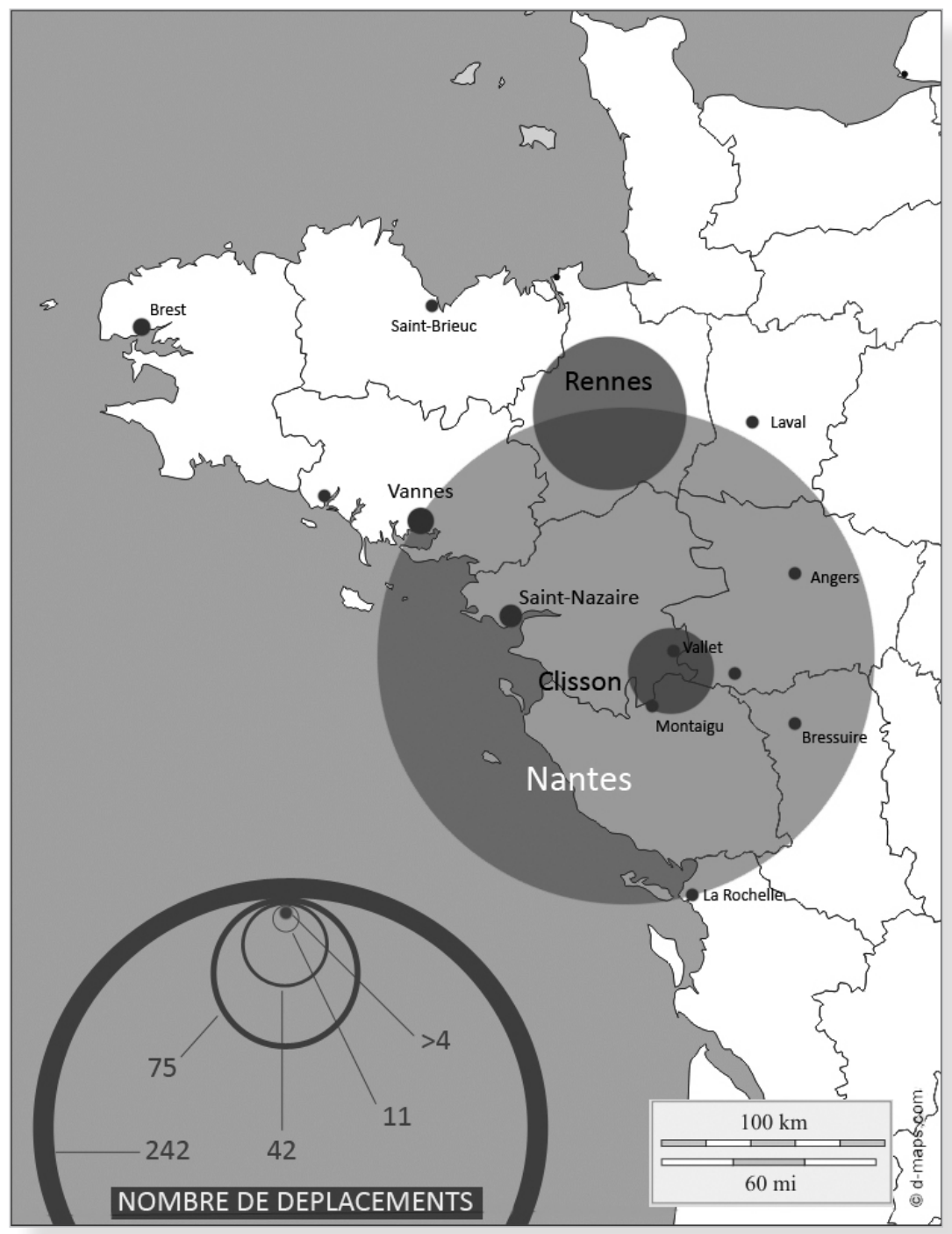

Figure 2

\section{Lorraine: déplacements au cours des 5 derniers concerts}

300 personnes spécialisé dans le rock et le metal situé en plein cœur de la ville de Nantes. À lui seul, le Ferrailleur rassemble $42 \%$ des concerts de metal nantais sur l'année étudiée ${ }^{21}$.

21. Ce qui représente une programmation de 54 concerts de metal entre juillet 2012 et juin 2013, c'est-à-dire 1 par semaine en moyenne rien que dans cette salle. 


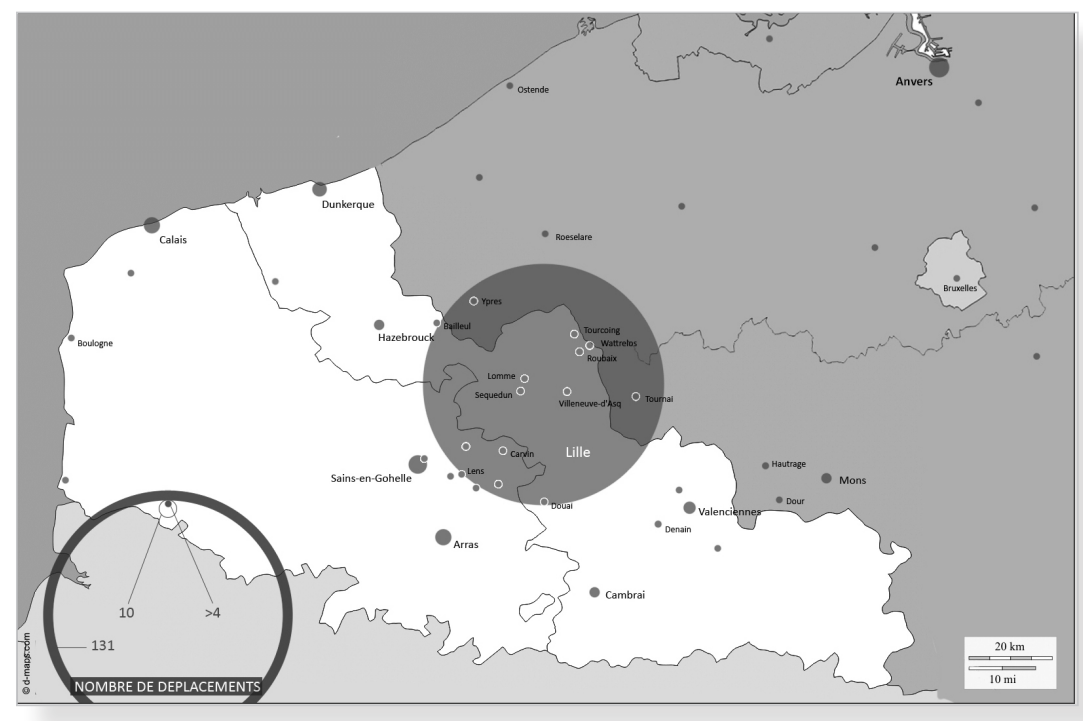

Figure 3

\section{Ille-et-Vilaine/Loire-Atlantique: déplacements au cours des 5 derniers concerts}

À ce propos, les amateurs de metal issus des départements d'Ille-etVilaine et de Loire-Atlantique interrogés au cours de l'enquête ont très largement fait part de leur perception ambivalente des deux villes de Rennes et de Nantes, et du rôle structurant que jouent les cafés-concerts qu'elles abritent, dans le dynamisme de la scène locale, comme semble l'illustrer le commentaire de cette amatrice interrogée dans le cadre du questionnaire:

De toute façon, les concerts sont dans ces lieux là [Rennes et Nantes]. Pour moi qui ait vécu dans les deux villes, il est clair que Rennes est en train de perdre tout son milieu underground, au profit de Nantes. On est loin de la création du groupe Sade dans les années 1980... Les deux bars, le Barock et le Troll Farceur, ont fermé et l'âme métalleuse s'est pas mal dissipée. Le Mondo Bizarro à Rennes reste toujours sympa, mais est trop connoté punk. Au contraire, Nantes, par ses trois bars concerts que sont Le Floride, le Ferrailleur et la Scène Michelet a véritablement créé un trio parfait. Je pense d'ailleurs que le point fondamental est bien le bar. Le disquaire n'est plus le lieu de référence avec Internet, l'espace de rencontre premier est le bar-concert. Et c'est là qu'on voit que la fermeture de deux bars à Rennes casse toute la synergie. Plus de lieux de rencontre, plus de rencontres entre petits musiciens, plus de vitalité d'une scène, plus les acteurs associatifs sont faibles. (Amatrice de metal ${ }^{22}$ de 22 ans, résidant à Rennes)

22. Questionnaire anonyme. 
C'est ainsi le consensus autour de la valeur attachée à un certain nombre de lieux identifiés par les usagers des scènes locales comme des pôles centraux et structurants de leurs territoires musicaux qui permet de déterminer les «hauts lieux» des scènes locales. Selon la définition du «haut lieu» donnée par Debarbieux, il s'agirait d'un «lieu érigé délibérément et collectivement au statut de symbole d'un système de valeur territorial ${ }^{23}{ }^{»}$. La dimension symbolique du «haut lieu» pour la musique metal est ainsi particulièrement perceptible dans l'exemple du Ferrailleur à Nantes :

Nantes, c'est le paradis du métalleux. Il y a des affiches ici qu'il n'y aura nulle part ailleurs, dans tous les domaines du metal. Là par exemple, le dernier concert que j'ai fait, c'était au Ferrailleur. C'était quand même Havok et Angelus Apatrida et dans le domaine du thrash c'est quand même une grosse grosse date et il n'y en a pas beaucoup en France. (Rémy, 21 ans, Nantes)

À proximité de Nantes, la petite ville de Clisson est par ailleurs elle aussi devenue un «haut lieu» de la musique metal en France et en Europe, puisque c'est la ville qui accueille chaque année le plus grand festival de metal en France, le Hellfest. Clisson est ainsi devenue une petite ville connue de tous les amateurs français et surtout un emblème pour la scène metal nantaise, sur laquelle rejaillit la renommée de Clisson. Depuis l'implantation du Hellfest en 2004 et les nombreuses synergies locales que le festival a générées, Nantes a ainsi vu naître et se pérenniser un certain nombre de lieux de diffusion et d'associations spécialisées dans la musique metal qui contrastent de façon draconienne avec le vide musical que connaissait la ville dans ce domaine, quelques années auparavant:

Pour l'extérieur ça nous a crédibilisé. Parce qu'il ne faut pas oublier que dans le temps, ici, il n'y avait rien. [...] Mais c'est vrai que voilà, tout d'un coup, on est un peu le centre de la France quelque part, avec le Hellfest. (Karen, 40 ans, multi-activiste de la scène metal, Nantes)

\section{La scène locale comme construction territoriale au-delà des frontières}

Si les scènes locales semblent constituées de noyaux durs assez nets, l'observation de la répartition des déplacements autour de ces épicentres permet de dessiner les contours de la scène locale. À ce titre, ces résultats mettent en lumière les limites induites par l'utilisation du découpage territorial administratif dans le cadre de l'analyse de scènes culturelles.

Le cas de l'Ille-et-Vilaine/Loire-Atlantique est révélateur, en ce que la scène ne semble pas se construire dans le cadre des limites administratives régionales. En effet, si nous avons déjà évoqué le fait que les déplacements des amateurs ren-

23. Bernard Debarbieux, «Du haut lieu en général et du mont Blanc en particulier», L'Espace Géographique, $n^{\circ} 1$, 1993, p. 5-13. 
nais vers la ville de Nantes ne sont pas anecdotiques, on constate en revanche que les amateurs de metal issus des départements d'Ille-et-Vilaine et de Loire-Atlantique, appartenant chacun à une région distincte ${ }^{24}$, se déplacent moins souvent dans le reste de leur région respective que dans le reste de la France. En effet, si environ un déplacement sur cinq concerne des destinations situées à l'extérieur de ces deux départements, seulement un déplacement sur vingt-cinq concerne un concert ayant eu lieu dans le reste de la région bretonne ou dans le reste de la région des Pays-de-la-Loire. Si les pratiques ne semblent donc pas définies à l'intérieur d'un cadre régional, elles sont en revanche beaucoup plus concentrées au sein des deux villes de Nantes et de Rennes et de leurs alentours (voir fig. 2). De ce point de vue, la région ne semble donc pas particulièrement structurante dans la construction de l'espace vécu de la scène locale.

À l'inverse de l'Ille-et-Vilaine et de la Loire-Atlantique, plus enclavées dans l'ouest du territoire français, les régions du Nord-Pas-de-Calais et de la Lorraine sont limitrophes à la Belgique, et la Lorraine possède en plus des frontières communes avec l'Allemagne et le Luxembourg. Cependant, ici encore, ces configurations transfrontalières viennent renforcer la faiblesse des limites régionales en tant que cadre des scènes metal locales. Ainsi, les publics du Nord-Pas-de-Calais se tournent volontiers vers les frontières belges pour se rendre en concert: plus d'un quart des amateurs (26\%) ont déclaré s'être rendu au moins une fois en Belgique pour assister à un concert de metal au cours des six derniers mois ${ }^{25}$. Les destinations belges sont diversifiées et concernent des salles de tailles variables, allant du petit café-concert à Tournai (le Cornwall, bar à thématique celtique) situé à 28 $\mathrm{km}$ de Lille, aux grands complexes destinés à la diffusion de musiques amplifiées (l'Ancienne Belgique à Bruxelles, $118 \mathrm{~km}$ de Lille, ou le Trix à Anvers, $125 \mathrm{~km}$ de Lille). La diversité des lieux cités en Belgique dans les résultats du questionnaire révèle des pratiques différenciées entre chaque individu, mais néanmoins une certaine familiarité avec le pays voisin. Il est ainsi difficile de désigner des «hauts lieux» belges pour la musique metal, bien que les déplacements en Belgique soient vécus par les amateurs comme une pratique ancrée dans leurs habitudes culturelles et récréatives.

Ce constat est similaire en Lorraine, où près d'un tiers des amateurs de metal $(31 \%)$ se sont rendus au moins une fois dans l'un des trois pays frontaliers pour assister à un concert de metal au cours des six derniers mois. Le public lorrain semble cependant avoir des habitudes plus marquées à

24. L'Ille-et-Vilaine appartient à la région Bretagne et la Loire-Atlantique au Pays de la Loire.

25. Alors que les autres déplacements à l'étranger cités par les enquêtés sont anecdotiques dans la mesure où ils représentent $3 \%$ des déplacements pour les amateurs d'llle-et-Vilaine et Loire-Atlantique, et les amateurs de Lorraine, et $4 \%$ pour les amateurs du Nord-Pas-de-Calais. Parmi ces villes étrangères citées, aucune ne concentre plus de $1 \%$ des déplacements. 


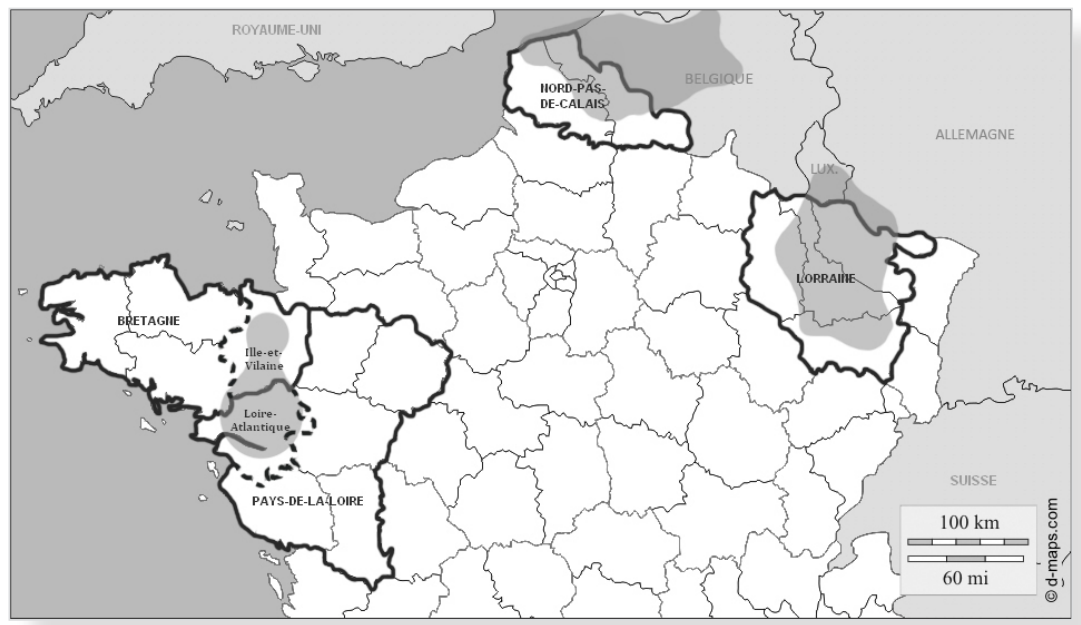

Figure 4

Représentation de l'«espace vécu» des scènes locales

l'étranger, où quelques salles allemandes et luxembourgeoises se distinguent nettement. C'est le cas de la Kulturfabrik d'Esch-sur-Alzette ${ }^{26}$ (ville frontalière luxembourgeoise), de l'Atelier à Luxembourg-ville (capitale du Luxembourg) et du Garage à Sarrebruck (ville frontalière allemande). Ce sont des salles de 500 à 2000 places, qui disposent chacune d'équipements adaptés à la diffusion des musiques amplifiées, mais aussi d'équipes de programmation sensibles aux esthétiques des musiques metal, et qui entretiennent des liens privilégiés avec les réseaux de booking spécialisés. Ces salles parviennent ainsi à capter des groupes de stature internationale, mais qui s'adressent néanmoins à des publics de niche et dont le passage est plus rare en Lorraine. Étant donné leur facilité d'accès par les réseaux routiers, ces lieux font ainsi partie intégrante de la scène locale vécue par les amateurs de metal lorrains.

Par ailleurs, il faut noter que la fréquentation des salles allemandes par le public lorrain l'amène également à côtoyer dans ces concerts un grand nombre d'autres pratiquants avec lesquels il ne partage pas forcément la même langue. Cette situation permet tout particulièrement d'observer la construction d'un sentiment d'appartenance à l'espace communautaire de la scène locale lorraine, dans le sens où la reconnaissance entre pairs (membres de la même scène locale) est amplifiée par le contexte étranger. 


\section{Perceptions de l'espace territorial recomposé par la musique}

\section{Niveaux d'éclatement}

Il s'agit ici de se focaliser sur les résultats d'une autre question posée aux amateurs: celui de la désignation de leur principale destination pour les concerts de metal. Les résultats obtenus à cette question, bien qu'ils viennent amplifier ceux déjà présentés, permettent d'apporter un nouvel éclairage. Ici, le point de vue est davantage celui des perceptions subjectives de l'espace territorial, plutôt que celui qui se rapporte aux déplacements effectivement réalisés au cours des derniers mois. Demander aux enquêtés quelle est la ville dans laquelle ils se rendent le plus souvent pour assister à un concert de metal, en excluant les déplacements anecdotiques qui peuvent avoir été réalisés récemment, mais qui ne leur semblent pas pour autant centraux ou représentatifs dans leurs pratiques au long cours, permet de vérifier s'il y a ou non un consensus autour d'un épicentre de la scène locale.

Si l'on s'efforce donc de repérer un enracinement majeur pour chaque scène vécue étudiée en s'intéressant à la désignation pour chaque enquêté d'une destination exclusive, il existe des similitudes frappantes entre l'emprise spatiale effective des individus issus des trois scènes locales et l'espace vécu a priori, avec le plus d'intensité. Ainsi, on retrouve les villes de Lille (77\% des réponses) pour les amateurs du Nord de la France, Nantes (64\%) et Rennes (23\%) pour les amateurs de l'Ouest, et Nancy (46\%) pour ceux de l'Est, citées comme destinations principales. Ces résultats viennent donc renforcer la désignation d'un épicentre pour chaque scène locale, qui s'avère bel et bien bipolaire dans le cas de la construction d'une scène locale à l'ouest du pays, partagée entre les départements d'Ille-et-Vilaine et de Loire-Atlantique. En effet, si Nantes possède toujours une nette avance sur la ville de Rennes, il devient ici assez clair que cette dernière reste identifiée par les amateurs comme un pôle structurant et emblématique de leur scène vécue.

De plus, si l'on observe le nombre de destinations citées pour chaque scène, on s'aperçoit que si les amateurs d'Ille-et-Vilaine/Loire-Atlantique n’ont cité que 6 villes différentes, et les amateurs du Nord-Pas-de-Calais, 9 villes différentes, les amateurs issus de la région lorraine ont en revanche cité 26 destinations principales différentes. Les réponses lorraines semblent donc traduire une plus grande multiplicité de points de vue sur leur scène locale, et notamment sur le choix de son épicentre. Outre Nancy, Esch-sur-Alzette au Luxembourg est par exemple citée comme destination principale par $13 \%$ des amateurs lorrains. En ce qui concerne le reste des réponses, $41 \%$ des 
répondants ont donc déterminé leur attachement principal à 24 autres lieux différents.

Ces résultats traduisent ainsi un éclatement beaucoup plus important en Lorraine que dans les deux autres régions dans la perception d'une cohérence de la scène locale organisée autour d'un pôle central, en ce que les publics lorrains semblent avoir une perception de leurs territoires musicaux très contrastée. À l'inverse, on a dans le Nord-Pas-de-Calais et la scène d'Illeet-Vilaine/Loire Atlantique des pôles d'attractivité beaucoup plus denses, avec des pratiques plus homogènes. Il semble donc y avoir un consensus beaucoup plus net dans ces deux régions sur la perception territoriale de la scène locale et de ses «hauts lieux».

Pour autant, on peut se demander si malgré l'apparente homogénéité des pratiques dominantes au sein de ces deux dernières scènes, la construction de cette cohérence collective perdure dans la singularité des pratiques individuelles.

\section{Variation des points de vue individuels}

Il s'agit ici de présenter deux cartes mentales ${ }^{27}$ récoltées auprès d'amateurs de metal nantais qui illustrent particulièrement la pluralité des représentations individuelles et subjectives d'une même scène locale ${ }^{28}$.

Rémy (Fig. 5) a 21 ans, est sans emploi fixe et se déplace essentiellement à pied ou en tramway. L'essentiel des concerts auxquels il participe ont lieu à Nantes, et bien qu'il avoue se déplacer à Rennes à l'occasion de quelques concerts dans l'année, il ne ressent pas d'attachement particulier avec cette ville:

J'y vais très peu, alors que c'est juste à côté. Mais généralement, j’y vais pour un concert, peut-être deux fois dans l'année. Par contre j'ai pas mal de copains qui sont, et sur Rennes, et sur Nantes, et qui font l'aller-retour Rennes-Nantes tout le temps. Moi je n'aime pas trop cette ville, personnellement. [...] L'ambiance n'y est pas forcément au rendez-vous.

Sur la carte mentale, c'est donc Nantes qu'il dessine. Son domicile actuel est représenté par une croix en bas de la carte et il fait aussi apparaître

27. Bob Rowntree, «Les cartes mentales, outil géographique pour la connaissance urbaine. Le cas d'Angers (Maineet-Loire)», Norois, n¹76, 1997.

28. Dessinées par les enquêtés à l'occasion d'entretiens réalisés au cours de l'enquête de terrain et contactés grâce au questionnaire, ces cartes représentent les endroits les plus souvent fréquentés par l'enquêté pour assister à des concerts de metal (et non forcément tous les lieux fréquentés). II s'agit donc ici de différentes représentations subjectives des «hauts-lieux» d'une scène locale. Le choix de ces lieux représente ainsi le point de vue réflexif des pratiques d'un seul individu à un moment «T» de sa carrière d'amateur. En effet, un lieu non mentionné sur la carte a pu être fréquenté par le passé, puis délaissé au profit d'autres lieux qui, eux, seront mentionnés sur la carte. De même que certains lieux fréquentés occasionnellement peuvent avoir été jugés trop anecdotiques par l'enquêté pour les faire apparaître sur sa carte. 


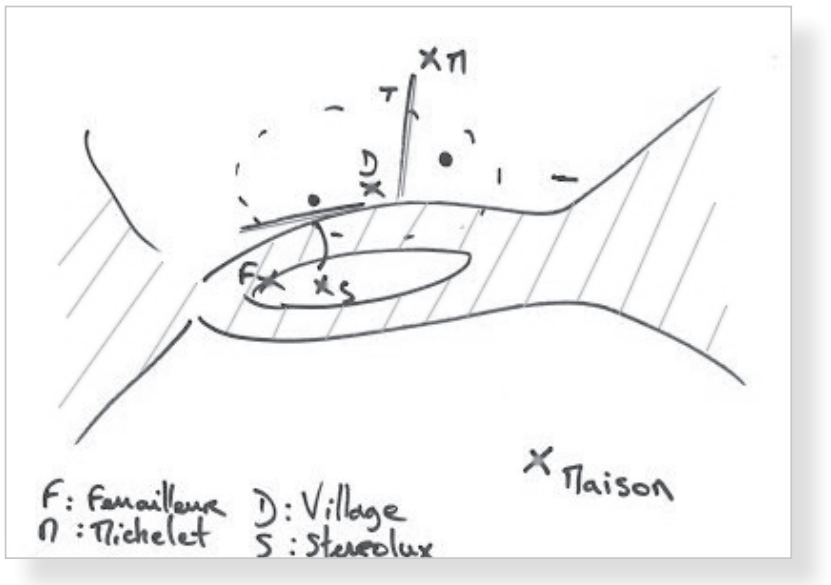

Figure 5

Carte mentale de Rémy, 21 ans, Nantes, Février 2014.

de façon très nette le fleuve de la Loire, qui sépare la ville en deux, et au milieu duquel se situe l'île de Nantes. Deux traits en angle droit représentent les deux lignes de tramway de la ville, qu'il emprunte notamment pour se rendre à la Scène Michelet, plus excentrée au Nord et située à proximité des universités. Il s'agit de l'un des trois cafés-concerts les plus actifs en termes de programmation metal à Nantes. Sont également représentés des lieux dont la programmation metal est plus anecdotique mais qu'il considère fréquenter régulièrement. Il s'agit du Stéréolux, une scène de musiques actuelles située sur l'île de Nantes à proximité duquel se trouve le Ferrailleur : «Le Ferrailleur, quand j'habitais près de la gare, il fallait vingt minutes pour y aller [à pied]. Ça se fait très bien». L'une des croix représente également un ensemble de lieux emblématiques de la vie culturelle nocturne nantaise, rassemblés dans un périmètre restreint du centre-ville et que les nantais nomment «Le Village». Ce quartier abrite notamment deux bars, le Café Death Porc et le De Dannan, qu'il décrit comme des «QG du metal à Nantes». Dans cette représentation subjective de son territoire musical vécu, les éléments topographiques de l'espace urbain semblent structurer assez nettement sa perception des lieux.

La seconde carte mentale (Fig. 6) est dessinée à une échelle radicalement différente: si Rémy avait dessiné une carte réduite à la ville de Nantes, sur la seconde, Julien a intégré les villes de Rennes et d'Angers.

Julien a 32 ans, un emploi, et réalise une grande partie de ses trajets en voiture. Sa représentation de la scène locale est structurée par trois métro- 


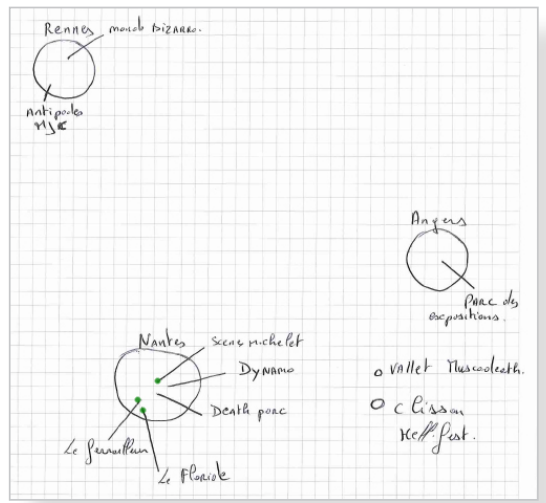

Figure 6

Carte mentale de Julien, 32 ans, Nantes, février 2014.

poles représentées par des ronds de taille similaire que sont Nantes, Rennes et Angers, qui est la troisième ville la plus peuplée du Grand Ouest après Nantes et Rennes. On retrouve sur sa carte trois lieux emblématiques de la scène metal nantaise déjà cités par Rémy (Le Ferrailleur, la Scène Michelet et le Café Death Porc), auxquels s'ajoute Le Floride, une boîte de nuit qui fait régulièrement office de café-concert quelques soirs par semaine, situé le long des quais désaffectés de l'île de Nantes et à proximité du Ferrailleur. Il s'agit de l'une des trois salles programmant le plus de concerts de metal à l'année à Nantes, à raison de 21 concerts sur l'année étudiée ${ }^{29}$. Le Dynamo, quant à lui, est un bar-concert situé à proximité du centre-ville de Nantes. À Rennes, on retrouve sur la carte le Mondo Bizarro, lieu alternatif et emblématique de la scène rock, punk et metal rennaise, situé sur une grande avenue excentrée du centre-ville. La $\mathrm{MJC}^{30}$ de l'Antipode est quant à elle un centre culturel produisant épisodiquement des concerts de metal ${ }^{31}$.

Julien a également représenté la ville d'Angers sur sa carte, pour y localiser le Parc des Expositions. Il s'agit d'un vaste complexe municipal dédié aux grandes manifestations et dôté d'une salle de spectacle à grande capacité d'accueil (9000 personnes). La ville d'Angers semble donc faire office d'un traitement particulier en ce qu'elle offre occasionnellement à l'enquêté la possibilité d'élargir l'offre de grands concerts à moins de $100 \mathrm{~km}$ de Nantes. Angers ne semble cependant pas fréquentée pour ses bars-concerts ou ses salles de proximité. Sont enfin représentées en bas à droite les grands festivals

29. Selon le relevé exhaustif de la programmation réalisé de juin 2012 à juillet 2013.

30. Acronyme pour Maison des Jeunes et de la Culture, qui sont des centres culturels associatifs dédiés à la jeunesse.

31. 6 concerts sur l'année étudiée. 
d'été auxquels il participe chaque année dans la région: le Hellfest à Clisson, ainsi qu'un petit festival de metal à Vallet, à proximité de Clisson: le Muscadeath, dont l'affiche fait régulièrement la part belle au groupes locaux et situé à 30 kilomètres au sud de Nantes.

La carte de Julien traduit donc une mobilité plus importante, et ainsi une perception de l'espace vécu de la scène locale bien plus vaste.

\section{Une mise en réseau de " hauts lieux» et d'espaces vides}

Les variations d'échelle et de point de vue permettent ainsi de rendre visibles les distances subjectives entre certains lieux, puisque, comme on l'a vu pour la Lorraine et les déplacements transfrontaliers, les pratiquants rapprochent également des lieux lointains à leur espace de vie, au même titre que n'importe quelle autre salle de proximité. La représentation que les pratiquants se font de leur scène locale a ainsi tendance à donner un sentiment de proximité avec le lointain «maîtrisé» et à produire des modes de construction de l'espace qui hiérarchisent les lieux entre eux: ceux qui sont pratiqués et représentés sur les cartes mentales par exemple, et l'ensemble des espaces vides qui peuvent s'interpréter comme autant de visions sélectives de l'espace géographique.

Les représentations mentales des scènes locales par leurs pratiquants semblent donc également ignorer de nombreux espaces géographiques, comme en Lorraine où le département de la Meuse n'est quasiment pas représenté, de même que de nombreux espaces compris entre Metz et Sarrebruck par exemple (Saint-Avold). Des salles tels que Le Garage en Allemagne ou la Kulturfabrik à Esch-sur-Alzette sont au contraire, et comme on l'a vu, reconnues comme des «haut-lieux» de la scène metal par le public lorrain. L'intégration de ces lieux au réseau de circulations de la scène lorraine par les pratiquants semble donc motivée par l'intérêt de leur programmation en comparaison à une offre localisée plus limitée et motivée par leur relative proximité, elle-même déterminée par leur facilité d'accès (réseaux autoroutiers).

La scène locale se construit donc sur l'articulation entre échelles sociales (l'individu, le groupe d'amis, la communauté de pairs) et échelles spatiales (le quartier, la ville, la région, le bassin de vie et les espaces de circulation). Cette articulation est ainsi rendue perceptible par le biais des circulations des acteurs locaux entre chaque lieu - soit des «entités socio-spatiales éclatées ${ }^{32}$ » - et de leur mise en relation au sein d'un réseau musical local.

32. Florence Boyer, «Échelle locale et mouvement: de la porosité spatiale et sociale dans les migrations circulaires», dans Elisabeth Boesen, Laurence Marfaing (dir.), Les nouveaux urbains dans l'espace Saharah Saël, un cosmopolisme par le bas, Paris, Éditions Karthala, 2007. 
Cette mise en réseau est rendue possible selon Jean-Pierre Augustin par la «mobilité accélérée», envisagée comme processus d'appropriation des espaces vécus, et qui favorise "un changement d'échelle urbaine remettant en question la distance physique comme indice de proximité sociale ${ }^{33}$.

Les représentations de l'espace des scènes locales se cristallisent ainsi autour d'un ensemble de «hauts lieux» vécus depuis l'intérieur par les usagers comme une mise en réseau sociologique et géographique de leurs espaces d'expérience, et parfois perçus depuis l'extérieur comme des éléments représentatifs de l'identité de la scène.

\section{Conclusion}

Il s'agissait donc de démontrer l'intérêt de sortir d'une vision en vase clos des scènes locales, pour s'intéresser de plus près aux déplacements de ceux qui les pratiquent. Utiliser cette approche a ainsi permis de montrer que dans le cas de ces trois scènes, la perception que les usagers se font de leur territoires musicaux est loin de correspondre aux limites administratives. Les scènes vécues par les usagers originaires de Lorraine et du Nord se construisent en effet très largement au-delà des frontières nationales, alors que la cohérence de la scène vécue dans la région Ouest semble se centraliser au sein de deux départements voisins qui appartiennent pourtant à deux régions administratives différentes.

À la lumière de ces résultats, il semble donc intéressant de reposer la question des limites géographiques d'une scène musicale ou culturelle. En effet, orienter le point de vue sur les déplacements des usagers permet de révéler la morphologie de l'espace vécu par les pratiquants et les modalités en fonction desquelles les individus ou les groupes d'individus construisent leur perception de la scène locale.

De même, malgré la multiplicité des points de vue sur la scène locale, c'est l'intensité des connexions entre les différents points du réseau, composé à la fois de personnes et de lieux (non nécessairement acteurs directs de la scène locale, comme c'était le cas dans l'exemple des bars sans concerts ou des «QG»), qui permet d'en révéler la cohérence et le niveau de structuration. Au sens de la sociologie de la traduction de Michel Callon, c'est donc le consensus autour des lieux emblématiques, malgré, comme on l'a vu, les variations d'échelles des réseaux individuels, qui permet de stabiliser la perception collective de la scène locale.

33. Jean-Pierre Augustin, Vincent Berdoulay, «Cultures vivantes, variations et créativités culturelles en région», SudOuest Européen, $n^{\circ} 8,2000$, p.1-4. 